\title{
Reporting COL7A1 gene mutations in five Iranian families with Dystrophic Epidermolysis Bollusa
}

\author{
Maryam Taghdiri ${ }^{1}$, Sirous Naeimi ${ }^{1}$, Majid Fardaei ${ }^{2}$, and Mohammad Bagher Tabei ${ }^{2}$ \\ ${ }^{1}$ Islamic Azad University Kazerun Branch \\ ${ }^{2}$ Shiraz University of Medical Sciences
}

April 28, 2020

\begin{abstract}
Dystrophic epidermolysis bullosa (DEB) is a inherited blistering disorder, characterized by affected dermis-epidermis conjunction. COL7A1 encodes collagen VII protein, which is responsible for dermis-epidermis conjunction. We report several mutations of COL7A1 in Iranian families, which result in DEB and expands the mutation spectrum of COL7A1 mutations for further molecular studies
\end{abstract}

Reporting COL7A1 gene mutations in five Iranian families with Dystrophic Epidermolysis Bollusa

Maryam Taghdiri ${ }^{1}$, Sirous Naeimi ${ }^{1 *}$, Majid Fardaei ${ }^{2}$, Seyed Mohammad Bagher Tabei ${ }^{2,3}$

1-Department of Genetics, Colleague of science, Kazerun branch, Islamic Azad University, Kazerun, Iran

2-Department of Medical Genetics, Shiraz University of Medical Sciences, Shiraz, Iran

3- Maternal-fetal Medicine Research Center, Shiraz University of Medical Sciences, Shiraz, Iran

*Corresponding Author: Sirous Naeimi, Department of Genetics, Colleague of science, Kazerun branch, Islamic Azad University, Kazerun, Iran

Email: naeimis@kau.ac.ir

\begin{abstract}
:
Dystrophic epidermolysis bullosa (DEB) is a rare inherited blistering disorder, characterized by affected dermis-epidermis conjunction. COL $7 A 1$ encodes collagen VII protein, which is responsible for dermis-epidermis conjunction. Here we report several mutations of COL7A1 in five Iranian families, which result in DEB and expands the mutation spectrum of COL7A1 mutations for further molecular studies.
\end{abstract}

Key Words: Skin disorder, Dystrophic Epidermolysis Bullosa, COL7A1, genetic mutations

Key Clinical Message: Different COL7A1 mutations in Iranian families expand the mutations spectrum for further molecular investigation and accurate genetic counseling in affected families.

\section{Introduction :}

Epidermolysis bullosa (EB) is a rare inherited skin fragility disorder, including fragile skin and mucous membrane as well as body blistering (1). Distinct mutations in the genes encoding cytoskeletal structure of keratinocytes or BMZ can cause EB. EB is classified into 4 main groups depending on clinical and molecular characteristics including EB simplex, dystrophic EB, junctional EB and kindler (2). 
Collagen VII is a major component of the anchoring fibrils which plays an important role in dermis and epidermis conjunction. Collagen VII is synthesized as a pro-collagen (pro- $\alpha 1$ ) in epidermal keratinocytes and dermal fibroblasts. Pro- $\alpha 1$ (VII) has triple helical central collagenous domain flanked by two noncollagenous globular domains (NC1and $\mathrm{NC2}$ ). $\mathrm{NC} 1$ is responsible for the attachment of anchoring fibrils at amino terminal and $\mathrm{NC} 2$ involves in antiparallel dimer formation at carboxyl terminal of collagen VII molecule. Gly-X-Y amino acid repetitions are located in the triple helical segment to assure its accurate conformation. These repeating sequences are disrupted 19 times by non-collagenous regions. These three identical pro- $\alpha 1$ (VII) chains assemble into type VII collagen homotrimers (COLVII) to ensure the dermalepidermal adhesion. The conservation of COL7A1 protein structure and amino acid sequences show type VII collagen importance as a structural protein $(3,4)$.

It was initially suggested COL7A1 gene as a candidate gene for dystrophic forms of EB (DEB) due to the structural modification of anchoring fibrils $(\mathrm{AFs})$ or great alterations in its numbers in a result of electron microscopy of the patients. COL7A1 gene (118 exons and 32kb on 3p21) encodes $\alpha 1$ chain of collagen protein VII. With the advancement of next generation sequencing a wide range of mutations were identified in COL7A1 gene. Nature and location of COL7A1mutations are strongly associated with the phenotypes. A large number of COL7A1 mutations are located in exon 73 and most of them can lead to premature termination codon (PTC).

Several domains of COL7A1 mediate interactions of macromolecules in the skin and interfere with skin integrity. The affected skin in DEB patients shows the sub-lamina densa cleavage at the level of (AFs) of the dermal-epidermal junction. DEB is a rare inherited blistering disorder with both autosomal recessive (RDEB) and autosomal dominant (DDEB) mode of inheritance.

The severe form of RDEB is primarily caused by premature termination codon (PTC) mutations, which can be attributed to bi-allelic nonsense, frameshift or splice site mutations. In this form, blisters expand through the body especially on the trauma-exposed sites such as fingers which leads to fingers fusion and seems as pseudosyndactyly. In the milder form of RDEB the fingers and nails are exposed to injury and is often resulting from compound heterozygous mutations (a PTC mutation on one allele and a missense mutation on another allele). In this condition, full length COL7A1 polypeptide with altered conformation can be synthesized and affects the stability of AFs structure. DDEB mutations usually involve glycine substitutions within the repeating Gly-X-Y sequences of triple helical domain. However, glycine substitutions were observed in either RDEB or DDEB depending on the position and nature of substitutions (4).

Biopsy of the latest blisters is a necessary process to find the depth of affected tissue and electron microscopy is a gold standard diagnostic procedure but it is not affordable. Immunofluorescence study and genetic mutation analysis are the effective way to identify and classify EB patients (1).

Here in this study, we report COL7A1 disease-causing mutations in five families affected by dystrophic epidermolysis bullosa disorder.

\section{Methods}

\subsection{Patient Reports}

Family I, patient I, II:

Two affected twin siblings were born to non-symptomatic consanguineous couple. A 32-yaer-old affected male selected genetic counseling to avoid his offspring inheriting the similar condition. He had complained about extensive blistering, skin fragility, digit fusion, pseudosyndactyly, fingers nail lost since childhood (fig1a). His twin sister had similar symptoms. Granulation tissue was observed on upper back of man but not her sister. Wounds were also observed on his elbows which biopsy revealed squamous cell carcinoma (fig1b). Mild mucosal involvement and oral blistering were clear in both cases and lead to malnutrition.

Family II, Patient III: 
The third patient was a 2-year-old girl who referred with severe scars on the skin and oral cavity, recurrent blistering, skin erosion, nail dystrophy and partial digit fusion of hands from the third months of life (Fig 2a). She was a product of full term vaginal delivery with normal weight and length and no sign of skin lesions. At the end of the second month, red scars appeared on the skin especially the abdomen, lower legs and face and also showed nail dystrophy. Small progressive blisters were observed all over the body. Severe blisters and joint contractures were noted in 1 years old of age. A skin biopsy revealed reduced numbers of anchoring fibrils and dermis-epidermis cleavage. There was positive family history of skin disorder in her cousins. Genetic investigation was conducted to evaluate current pregnancy for identified mutation.

\section{Family III, Patient IV}

A 6-year-old girl, product of consanguineous couple, presented with skin erosion, mild milia especially around eyelid, loss of nails, digit fusion, joint contractures and scars of oral cavity. Laboratory evaluations showed low hemoglobin levels due to anemia. Electron microscopy of wounds revealed hypoplastic anchoring fibrils and cleavage at the level of the sublamina densa, which were consistent with DEB. The family was referred to genetic counseling to identify causative gene mutation.

\section{Family IV, Patient V}

The patient was 4 years 6 months old boy, he was referred with skin fragility, mucous membrane involvements and severe itching on the hands and feet. Physical examination showed blisters on the knees and elbows, joint contractures, ophthalmic abnormalities, oral ulcers and teeth deformities. Sparse hair and eyebrows were also observed.

Typical laboratory evaluations revealed low hemoglobin and vitamin D. His uncle was also affected by skin disorder and died at 4 years of age. The patient was the first child of healthy consanguineous couple and was a product of full term normal vaginal delivery. Parents did not give consent to skin biopsy because of pain and catastrophic condition of their child. However, the clinical symptoms were consistent with EB. Therefore, genetic study was recommended to clarify the disease-causing mutation and evaluate current pregnancy for identified mutation.

Family V, Patient VI:

The patient was a 27-year-old male, the only affected child of twelve siblings born to non- consanguineous parents. Scattered red spot, mild scarring was observed on the upper back, hands and lower leg at birth. Most of his blisters were particularly limited to trauma-exposed areas and bony prominences. He had no eye, nail, digit, oral involvements. He complained skin itching and recurrent blisters at knees and elbows at the time of experiment. Transmission electron microscopy of skin biopsy revealed a cleavage beneath the lamina densa and absence of a large number of anchoring fibrils. On the basis of clinical symptoms and no family history of skin disorder, dominant form of DEB was considered for the patient and was referred to genetic counseling to evaluate the mutation.

\subsection{Genetic testing}

WES was performed on the parents of dead patients or on the available patients to capture and enrich all exons of COL7A1 gene in addition to other essential flanking parts. Next generation sequencing was carried out using Illumina Hiseq 2000 machine to sequence about 100 million reads and standard Illumina protocol for pair-end 99 nucleotide sequencing. Aligning reads, identifying and annotating genetic variants were carried out using BWA aligner, GATK and ANNOVAR as bioinformatics tools, respectively (5-7).

Sanger sequencing was done on all individuals in order to confirm the WES results and also to find the genotype of other family members. Whole blood samples were collected from participants of all seven families in EDTA tube. DNA was extracted from blood and CVS samples using QIAamp DNA Minikit (Qiagen, Germany) according to the manufacturer's instructions. DNA concentration was evaluated using NanoDrop C (Thermofisher, USA) and then kept at $-20^{*} \mathrm{C}$ until use. Table S1 shows primer pairs that were used to amplify the desired genome segment in each family. 
Sanger sequencing was then carried out in both forward and reverse directions on the amplified DNA using ABI BigDye Terminator Cycle Sequencing kit (Applied Biosystems ${ }^{\circledR}$, USA).

\section{Results :}

NGS data analysis of five families showed different variant in distinct part of COL7A1 gene.

In the first family a homozygous variant (COL7A1, NM_000094, c.5018G > A, p.G1673E) of unknown significant in COL7A1 gene was identified. The homozygous and heterozygous state of the mutation were confirmed by sanger sequencing in affected individuals (proband and his sister) and their parents, respectively. Therefore, this mutation is inherited as autosomal recessive pattern and mutation in this gene is associated with recessive dystrophic epidermolysis bullosa disorder (Fig 1c). According to mutation taster prediction (8), this glycine substitution variant is disease causing variant. Multiple sequence alignment using T-COFFE online software was performed across different animal kingdoms and confirmed the highly conservation of 1673 glycine amino acid (Fig 1d).

In family II, WES was performed on a 2-year-old girl who showed general DEB phenotypes. The results showed a homozygous mutation in COL7A1 gene (COL7A1, NM_000094, c.425A > G, p.K142R). The patients passed away due to accident at the time of confirmation and we didn't have access to her DNA sample. Therefore, sanger sequencing was performed only on parents and the heterozygous mutation confirmed in her parents, which might show the autosomal recessive mode of inheritance in this family. Sanger sequencing analysis of CVS sample had showed that the first pregnancy had a homozygous mutant variant and the fetus was affected. The second pregnancy was heterozygous and healthy. This missense variant was reported previously as a disease-causing mutation (rs121912856) which could give rise to RDEB (Fig 2b). Multiple sequence alignment was performed across different animal kingdoms and confirmed the highly conservation of lysine 142 (Fig 2c).

In family III, the NGS results revealed a novel homozygous, non-frameshift deletion and a frameshift insertion in exon 2 ofCOL7A1 gene (COL7A1, NM_000094: c.180_182del, p.60_61del \& c.179-180 ins GAAA, p.F60fs). This mutation was confirmed as homozygous in patient and heterozygous variant in healthy sibling and parents. The autosomal recessive pattern was confirmed for mentioned mutation in this family (Fig 3a).

This variant was predicted as disease-causing mutation by online mutation taster software. The insertion of 4 nucleotides (GAAA) results in frameshift mutation after position 60 in amino acid sequence.

Analysis of WES data of patient in family IV showed a deleterious homozygous splice site mutation in exon 6 of COL7A1 gene (COL7A1, NM_000094: c.682+1 G>A). The mutation previously was reported as a pathogenic variant. The mutation was confirmed in the parents by sanger sequencing. Heterozygous parents let us concluded the probable autosomal recessive mode of inheritance for mentioned mutation in the family (Fig3b).

In family $\mathrm{V}$, WES test showed glycine substitution mutation in exon 73 of COL7A1 gene (COL7A1, NM_000094: c.6101G>A, G2034E). Using sanger sequencing this heterozygous mutation was confirmed in proband. This mutation was not identified in parents, so this is considered as a de novo mutation with autosomal dominant mode of inheritance (Fig 4a).

Multiple sequence alignment using T-COFFE online software was done across distinct animal kingdoms and confirmed the highly conservation of 2034 glycine during evolution (Fig 4b).

\section{Discussion}

Different COL7A1 gene mutations result in dystrophic epidermolysis bullosa disorder. DEB patients are characterized by affected dermis-epidermis conjunction and they are extremely prone to blistering by mechanical stimulation. The affected genome location of DEB patients was localized on the short arm of chromosome 1 by genetic linkage analysis. This region encompasses COL7A1 gene which has 118 exons and span $32 \mathrm{~Kb}$ of human genome $(9,10)$. collagenVII is a homotrimer molecule consists of three identical $\alpha 1$ chains with various Gly-X-Y repetition. The glycine, with the single hydrogen atom in the side chain, is the smallest 
amino acid which provides the flexibility in collagen molecule by allowing flexible bending of the polypeptide. The Glycine substitution (GS) mutation could give rise to both autosomal recessive and dominant form of DEB (4).

Here, we report two families with GS mutations in different region of COL7A1 gene which were inherited in autosomal recessive mode in family I (COL7A1, NM_000094, c.5018G > A, p.G1673E) and autosomal dominant in family V (COL7A1, NM_000094: c.6101G>A, G2034E). Glycine has critical structural roles in the triple helical structure. The substitution of glycine for large amino acids might destabilize triple helix structure of collagen molecule. The recessive GS mutation inhibits $\alpha$-chain synthesis completely. According to protein details of UniProt database amino acids 1254-2784 constitute the triple helix region of the protein. The glycine 1673 and 2034 is located in this region, hence affect the triple helical structure. Glycine substitutions in collagen VII have adverse consequences on the formation and function of anchoring fibrils. Bruckner-Tuderman et al identified G2034R mutation interfering with protein folding and result in collagen VII accumulation (11).

According to the mutation taster prediction, c.5018G $>$ A mutation exerts its effect on splicing site by producing cryptic donor splicing site in exon 54 which affect downstream structures such as disulfide bond and NC2 region of the protein. In 2011, G1673R was reported as disease-causing substitution in DEB patients (12). Therefore, this mutation cause RDEB and is disease causing variant.

Another GS mutation was observed in family V. The p.G2034E substitution was reported as a disease-causing mutation in dominant mode (13). Here, the results also revealed heterozygous mutation in patient. However, nor mother neither father had this mutant allele. Hence, this mutation was inherited as autosomal dominant manner and it seemed that G2034E was a de novo event due to no family history of EB. In addition, this mutation can affect downstream structure by producing cryptic donor splice site mutation.

Mild DEB patient without any positive family history usually were known as de novo or sporadic DDEB cases. De novo individuals often had dominant mutation in COL $7 A 1$ and transfer the mutation to offspring with $50 \%$ probability. Various missense mutations of this region or flanking segments are reported in DEB patients which showed the importance of this region of type VII collagen molecule (4). De novo GS leading to DDEB has been reported in the following positions:

G1775D (14), G2012S (15), G2012D (16), G2028R (17), G2034R ,G2034W(18), G2040V (19), G2067R (14), G2076D (20), G2079E (21),G2348R (22).

Substitution of glycine in 2034 position for arginine and tryptophan amino acids were previously reported in affected individuals (18) and in this study G2034E was identified in our patient.

The deleterious mutation of family II (COL7A1, NM_000094, c.425A>G, p.K142R) was previously reported as the first mutation which leads to splicing deficiency of COL7A1. It is located in the vicinity of the donor splice site (position -2 of the intron 3) which produce three aberrant mRNA depending on the skipping of exon 3, activation of cryptic donor splice site within exon 3 or retention of entire intron 3. Gardella, et al identified a premature termination codon (PTC) before the end of exon 4 in all altered transcripts resulting in truncated pro- $\alpha 1$ (VII) chains. This chains are likely to degrade by nonsense mediated decay (NMD) mechanism and do not include in COLVII anchoring fibrils (23). Nonsense-mediated decay (NMD) is the mRNA surveillance mechanism which degrades mRNA with in-frame PTC to protect cells from dominant negative effect (24).

Haplotype analysis of $C O L 7 A 1$ gene have been identified some recurrent mutations in distinct patients with similar allelic background. c.425A $>\mathrm{G}$ mutation was found in Italy and central European cases as recurrent one (4).Lysine 142 is a conserved amino acid during evolution and its mutation has an adverse effect on splicing site and protein synthesis. Mutation taster database uses a third party splice site prediction online software, called NNsplice to analyze possible changes in splice sites. According to this analysis, this mutation is likely to disturb normal splicing by omitting donor site of intron 3 (exon-intron border: wt:CAAG/gtga, mu: CAGG/gtga) and results in sequence motif lost. 
Protein details of UniProt database revealed that this amino acid is located in NC1 non-collagenous domain which is responsible for the attachment of anchoring fibrils at amino terminal of collagen VII.

Another splicing site point mutation (COL7A1, NM_000094: c.682+1 G>A) was identified in family IV which was previously reported as compound heterozygous with p.R578X mutation. Additionally, a compound heterozygous $682+1 \mathrm{G}>\mathrm{C}$ and GS mutation showed severe phenotypes which indicates expression reduction due to splice site mutation and exon 5 omission $(4,25)$.

Point mutation in highly conserved nucleotides such as GT at 5' end and AG dinucleotide at 3' end of an intron have a detrimental effect on RNA splicing with various deleterious effects on transcription, including exon skipping, intron retention or frameshift mutation which might result in in-frame PTC and NMD (24).

Non frameshift deletion and frameshift insertion (COL7A1, NM_000094:c.180-182del.p.60-61del \& c.179180insGAAA,p.F60Fs) were detected in family III. The identified mutation in exon 2 of COL7A1 seems pathogenic in this extremely gene.

The conservation values of the nucleotides in this region is 1 in phastcons and 3.85 in phylop. Phastcon online software evaluate the given nucleotide conservation based on the multiple sequence alignment across genome sequences of 46 different species (The values vary between 0 and 1). The Values close to 1 reflect the high probability of conservation. Phylop measure conservation as well as acceleration of the evolution. Positive scores revealed conserved sites and negative scores predict fast-evolving sites.

The wild type amino acid of stop codon is at 2945 while the mutant one is at amino acid 88 . Therefore, frameshift mutation could indirectly lead to in-frame PTC and ultimately produces truncated proteins that might have the potential to interfere the function of normal protein by dominant negative effect. In this family the mutation give rise to extremely short protein and NMD seems to be crucial to protect cells.

The identified mutations improve scientific knowledge of the EB causative mutations, which facilitates the EB classification to consider effective treatment and appropriate ways to take care of patients. Moreover, these genetic data will help to investigate the frequency of these mutations in other affected Iranian families and perform genetic counselling accurately.

\section{Conflict of interests}

The authors declare that there are no competing interests

\section{Author contribution}

MT clinically evaluated the patient, did the genetic studies, drafted the manuscript. SN, MF, SMT designed the study and revised the manuscript.

\section{Acknowledgments}

We would like to thank all patients and families for their willingness to take part in this study. We would like to thank Ms. Ghazale Mahjoub for her help in current paper and performing some relevant bioinformatics analysis.

Figure Legends

Figure 1: Family I. A) Patient I, pseudosyndactyly is clear in the photo B) Wound on elbow of the patient I which leads to squamous cell carcinoma. C) pedigree and sequence chromatograms. Both parents are heterozygous and the affected twin are homozygous for the identified glycine substitution mutation. D) Comparative amino acid alignment shows the conservation of amino acid glycine 1673 across distinct animal kingdoms. Symbols: $\left(^{*}\right)$ identical amino acids; (:) just similar amino acids.

Figure 2: Family II, A) patient III, Hand and neck wounds are clear in the picture. B) Pedigree and sequence chromatograms. Both parents are heterozygous for identified mutation. The mutation was confirmed in the proband as homozygous. The first pregnancy was homozygous and affected and the second one was carrier 
heterozygous and healthy. C) Comparative amino acid alignment shows the conservation of amino acid lysine 142 across distinct animal kingdoms. Symbols: (*) identical amino acids; (:) just similar amino acids.

Figure 3: A) Family III Pedigree and sequence chromatograms. The proband was homozygous for identified mutation and her parents and sister were carrier heterozygous of this mutation in COL7A1 gene. B) Family IV pedigree and sequence chromatograms. Both parents are heterozygous for splice site mutation in COL7A1 gene.

Figure 4: Family V, A) Pedigree and sequence chromatograms. Both parents didn't reveal the mutation, while it was heterozygous in proband, which indicates the autosomal dominant inheritance. B) Comparative amino acid alignment shows the conservation of amino acid glycine 2034 across distinct animal kingdoms during evolution. Symbols: (*) identical amino acids; (:) just similar amino acids.

\section{References}

1. Yadav RS, Jayswal A, Shrestha S, Gupta SK, Paudel U. Dystrophic Epidermolysis Bullosa. JNMA; journal of the Nepal Medical Association. 2018;56(213):879-82.

2. Boeira VL, Souza ES, Rocha Bde O, Oliveira PD, Oliveira Mde F, Rego VR, et al. Inherited epidermolysis bullosa: clinical and therapeutic aspects. An Bras Dermatol. 2013;88(2):185-98.

3. Has C, Nystrom A, Saeidian AH, Bruckner-Tuderman L, Uitto J. Epidermolysis bullosa: Molecular pathology of connective tissue components in the cutaneous basement membrane zone. Matrix biology : journal of the International Society for Matrix Biology. 2018;71-72:313-29.

4. Dang N, Murrell DF. Mutation analysis and characterization of COL7A1 mutations in dystrophic epidermolysis bullosa. Experimental dermatology. 2008;17(7):553-68.

5. Li H, Durbin R. Fast and accurate short read alignment with Burrows-Wheeler transform. Bioinformatics (Oxford, England). 2009;25(14):1754-60.

6. McKenna A, Hanna M, Banks E, Sivachenko A, Cibulskis K, Kernytsky A, et al. The Genome Analysis Toolkit: a MapReduce framework for analyzing next-generation DNA sequencing data. Genome research. 2010;20(9):1297-303.

7. Wang K, Li M, Hakonarson H. ANNOVAR: functional annotation of genetic variants from high-throughput sequencing data. Nucleic acids research. 2010;38(16):e164.

8. Schwarz JM, Cooper DN, Schuelke M, Seelow D. MutationTaster2: mutation prediction for the deepsequencing age. Nature Methods. 2014;11(4):361-2.

9. Ryynanen M, Ryynanen J, Sollberg S, Iozzo RV, Knowlton RG, Uitto J. Genetic linkage of type VII collagen (COL7A1) to dominant dystrophic epidermolysis bullosa in families with abnormal anchoring fibrils. The Journal of clinical investigation. 1992;89(3):974-80.

10. Greenspan DS, Byers MG, Eddy RL, Hoffman GG, Shows TB. Localization of the human collagen gene COL7A1 to 3p21.3 by fluorescence in situ hybridization. Cytogenetic and Genome Research. 1993;62(1):35-6.

11. Bruckner-Tuderman L, Hopfner B, Hammami-Hauasli N. Biology of anchoring fibrils: lessons from dystrophic epidermolysis bullosa. Matrix biology : journal of the International Society for Matrix Biology. 1999;18(1):43-54.

12. Frew J, Lim SW, Klausseger A, Chow CW, Tran K, Su J, et al. Autosomal dominant bullous dermolysis of the newborn associated with a heterozygous missense mutation p.G1673R in type VII collagen. The Australasian journal of dermatology. 2011;52(4):e1-4.

13. Kern JS, Kohlhase J, Bruckner-Tuderman L, Has C. Expanding the COL7A1 mutation database: novel and recurrent mutations and unusual genotype-phenotype constellations in 41 patients with dystrophic epidermolysis bullosa. The Journal of investigative dermatology. 2006;126(5):1006-12. 
14. Posteraro P, Pascucci M, Colombi M, Barlati S, Giannetti A, Paradisi M, et al. Denaturing HPLC-based approach for detection of COL7A1 gene mutations causing dystrophic epidermolysis bullosa. Biochemical and biophysical research communications. 2005;338(3):1391-401.

15. Gardella R, Castiglia D, Posteraro P, Bernardini S, Zoppi N, Paradisi M, et al. Genotype-phenotype correlation in italian patients with dystrophic epidermolysis bullosa. The Journal of investigative dermatology. 2002;119(6):1456-62.

16. Matsuba S, Suga Y, Mayuzumi N, Ikeda S, Ogawa H. A Japanese case of de novo dominant dystrophic epidermolysis bullosa. Clinical and experimental dermatology. 2002;27(1):56-8.

17. Murata T, Masunaga T, Shimizu H, Takizawa Y, Ishiko A, Hatta N, et al. Glycine substitution mutations by different amino acids in the same codon of COL7A1 lead to heterogeneous clinical phenotypes of dominant dystrophic epidermolysis bullosa. Archives of dermatological research. 2000;292(10):477-81.

18. Mecklenbeck S, Hammami-Hauasli N, Hopfner B, Schumann H, Kramer A, Kuster W, et al. Clustering of COL7A1 mutations in exon 73: implications for mutation analysis in dystrophic epidermolysis bullosa. The Journal of investigative dermatology. 1999;112(3):398-400.

19. Rouan F, Pulkkinen L, Jonkman MF, Bauer JW, Cserhalmi-Friedman PB, Christiano AM, et al. Novel and de novo glycine substitution mutations in the type VII collagen gene (COL7A1) in dystrophic epidermolysis bullosa: implications for genetic counseling. The Journal of investigative dermatology. 1998;111(6):12103 .

20. Kon A, Nomura K, Pulkkinen L, Sawamura D, Hashimoto I, Uitto J. Novel glycine substitution mutations in COL7A1 reveal that the Pasini and Cockayne-Touraine variants of dominant dystrophic epidermolysis bullosa are allelic. The Journal of investigative dermatology. 1997;109(5):684-7.

21. Kon A, McGrath JA, Pulkkinen L, Nomura K, Nakamura T, Maekawa Y, et al. Glycine substitution mutations in the type VII collagen gene (COL7A1) in dystrophic epidermolysis bullosa: implications for genetic counseling. The Journal of investigative dermatology. 1997;108(2):224-8.

22. Cserhalmi-Friedman PB, Grossman J, Karpati S, Ahmad W, Horvath A, Christiano AM. Identification of a de novo glycine substitution in the type VII collagen gene in a proband with mild dystrophic epidermolysis bullosa. Experimental dermatology. 1999;8(2):143-5.

23. Gardella R, Belletti L, Zoppi N, Marini D, Barlati S, Colombi M. Identification of two splicing mutations in the collagen type VII gene (COL7A1) of a patient affected by the localisata variant of recessive dystrophic epidermolysis bullosa. American journal of human genetics. 1996;59(2):292-300.

24. Strachan T, Goodship J, Chinnery P. Genetics and genomics in medicine: Taylor \& Francis; 2014.

25. Drera B, Ritelli M, Tadini G, Zoppi N, Venturini M, Calzavara-Pinton PG, et al. The novel p.G150R missense mutation in the cartilage matrix protein subdomain of type VII collagen in compound heterozigosity with the c. $682+1 \mathrm{G}>$ A COL7A1 splicing mutation leads to mild dystrophic epidermolysis bullosa. Journal of dermatological science. 2009;53(3):222-5. 

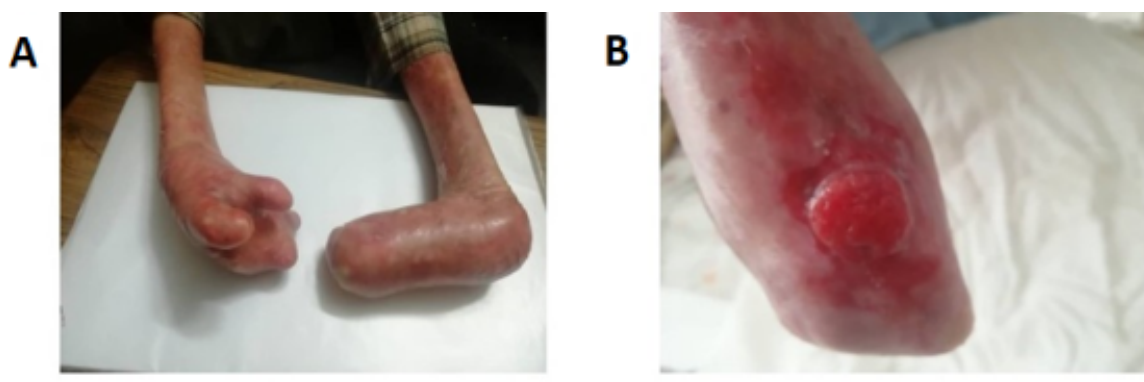

C G T C T Y

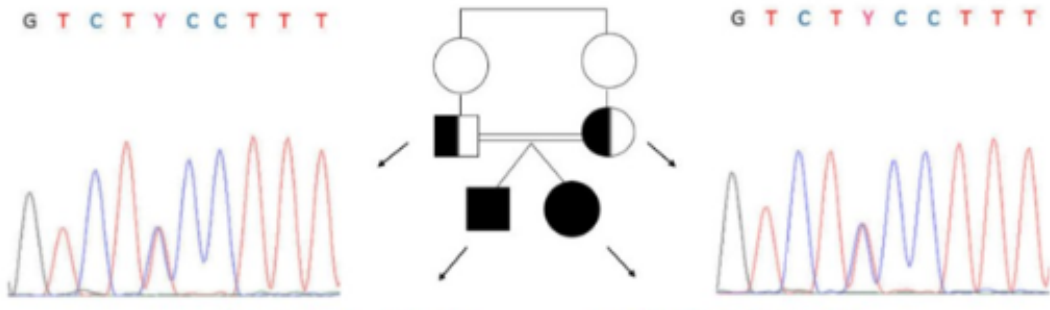

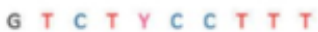

G T C T T C C T T T

G T C T T C C T T T
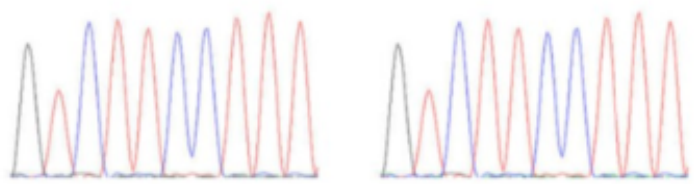

D

Zebrafish GDTGDPEDGRNGTPGTMGPRGPKGDQGIQGPPGPPGDVTTGEKI

Mouse

Elephant

Dog GDQGDPGEDGRNGSPGSSG -..-PKGDRGEPGPPGPPGRLVDAGI GDQGDPGEDGRNGSPGPSG - - - PKGDRGEPGPPGPPGRLVDTGL GDQGDPGEDGRNGSPGPSG -..PKGDRGEPGPPGLPGRLVDSGL GDOGDPGEDGRNGSPGPSG ... PKGDRGEPGPPGPPGRLVDTGP

Gorilla

Human

GDQGDPGEDGRNGSPGPSG - . - PKGDRGEPGPPGPPGRLVDTGP

Chimpanzee

GDQGDPGEDGRNGSPGSSG - - PKGDRGEPGPPGPPGRLVDTGP

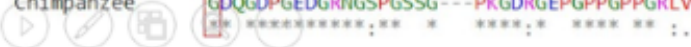
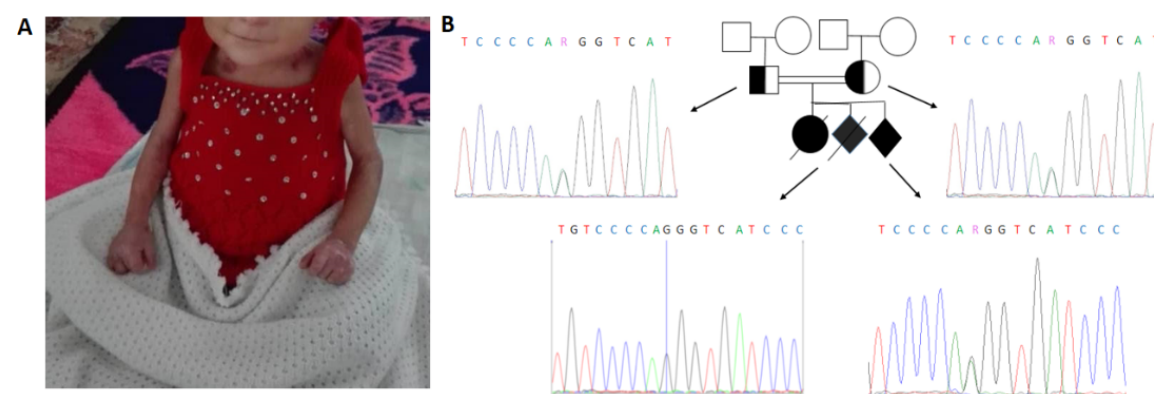

C

Zebrafish

Mouse

Elephant

Dog

Gorilla

Human

Chimpanzee
NTRTGAGLKYIADNFFSPASI-RDVPTKISILITDGKSQDNVLEPSQKLRGLG' NTRTGAALHHVSDRVFLPRLTRPGVPKNCILITDGKSQDLVDTAAQKLKGQG NTRTGAAILHVADRIFLPQLARPGIPKNCILITDGKSQDFVDTAAQRLKGQG NTRTGAAILHVADHVFLPQLARPGVPKNCILITDGKSQDLVDTAAQRLKGQG NTRTGAAILHVADHVFLPQLARPGVPKNCILITDGKSODLVDTAAQRLKGQG' NTRTGAAILHVADHVFLPQLARPGVPKNCILITDGKSQDLVDTAAQRLKGQG NTRTGAAILHVADHVFLPQLARPGVPKNCILITDGKSQDLVDTAAQRLKGQG 
A

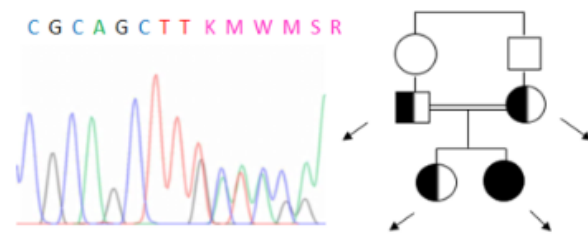

C G C A G C T TKM W M S R

C A $\quad G \quad C \quad T \quad T \quad K M \quad M \quad M \quad R$

C A G C T T G A A A C
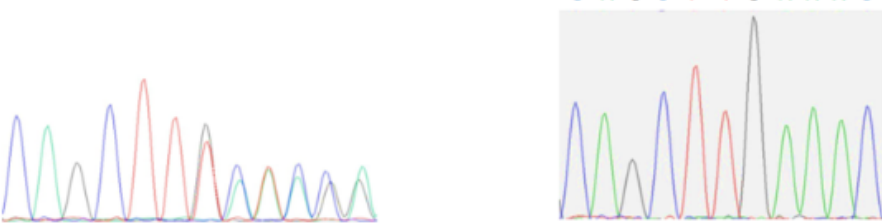

B
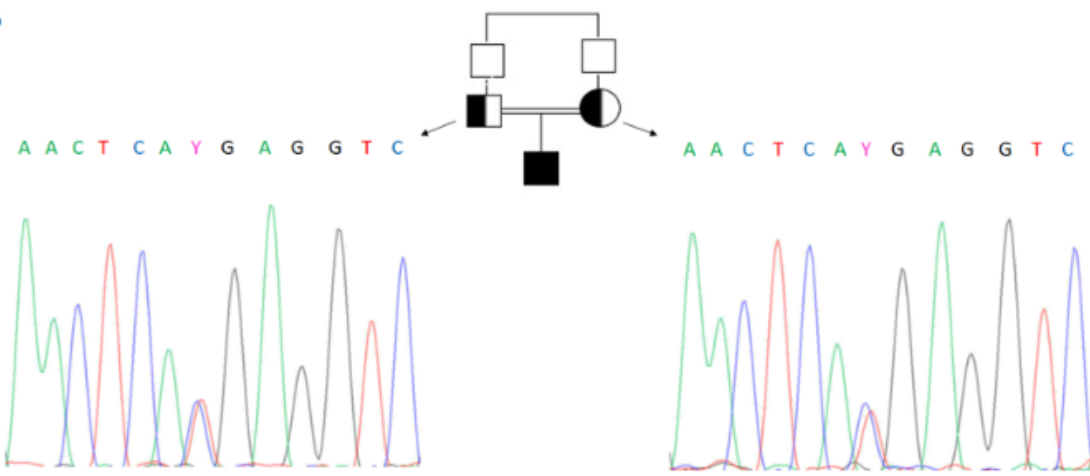

A
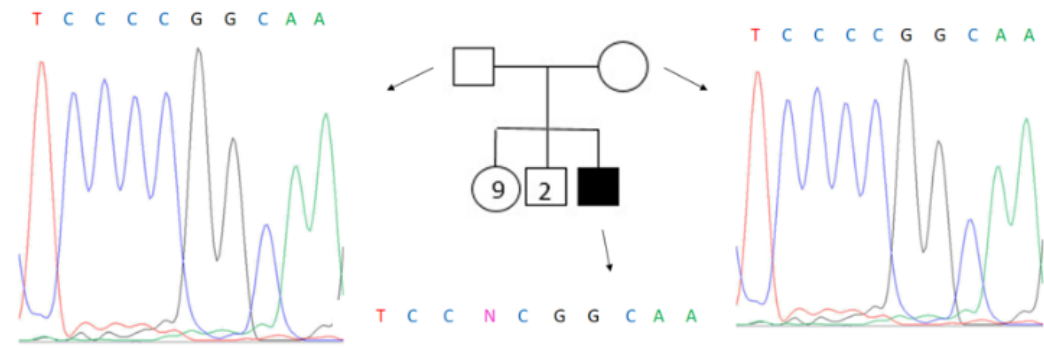

B

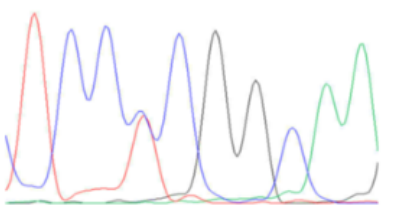

Zebrafish

Mouse

Elephant

Dog

Gorilla

Human

Chimpanzee

GLPGERGMKGEQGEKGPAGPQGPTGRAIGERGPEGPPGQAFEEPGKPGIPGVPGRAGELGE GFPGERGLKGERGD - - PGPQGPPGLALGERGPPGPPGLAGEPGKPGIPGLPGRAGGSGE GFPGERGLKGDRGD- - -PGPQGPPGLALGERGLPGPPGLAGEPGKPGIPGLPGRAGSVGE GFSGERGLKGERGD - - PGPQGPPGLALGERGPPGPPGLAGEPGKPGIPGLPGRAGGAGE GFPGERGLKGDRGD - - -PGPQGPPGLALGERGPPGPSGLAGEPGKPGIPGLPGRAGGVGE GFPGERGLKGDRGD- - - PGPQGPPGLALGERGPPGPSGLAGEPGKPGIPGLPGRAGGVGE GFPGERGLKGDRGD---PGPQGPPGLALGERGPPGPSGLAGEPGKPGIPGLPGRAGGVGE

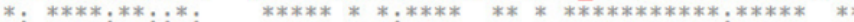

